\title{
Denominational Composition of the Kievan Nobility 1569-1648
}

In the discussion and comparisons presented below, different methods have been applied to analyse particular social layers of the nobility. The problem of religious changes that occurred in knyaz families has been studied in detail and thus it will not be addressed here. ${ }^{1}$ Due to the lack of relevant sources, also the boyar class will not be discussed. I will, however, try to present the denominational composition of Ruthenian nobility and gentry. In the present state of research the analysis of noble families can be much more detailed than it is possible in the case of gentry. In this case I attempt to determine the denomination of all adult members of the families being active in the period under discussion which is a base for a statistical analysis. In the case of gentry families only fragmentary data, related to a limited number of persons, is analysed. To supplement it, I attach also information on the denomination of members of the most important incoming Polish and Lithuanian families.

Sources and bibliography

Any research on the denominational composition of the nobility in the incorporated voivodeships relates to a specific set of sources. The descriptive material - memoirs, diaries or correspondence - is used rather rarely,

1 Recently discussed by N. Jakovenko, 'Relihijni konversiji. Sproba pohljadu zseredyny', in eadem, Paralelnyj svit. Doslidžennya z istoriji ujavlen’ ta idej w Ukrajini XVI-XVII st., Kiev, 2002. 
because of its scarcity. ${ }^{2}$ When presenting a list of such sources, one should emphasise that some of them are particularly useful. An important source is the journal of an orthodox gentleman, Joachim Jerlicz. ${ }^{3}$ His chronicle gives an account of family and religious events (like christenings, weddings and funerals) that occurred in his social circle. This allows the denomination of many members of that group to be ascertained. The correspondence that survived is of lesser importance. A useful collection of sources is provided by the Basilian centre in Rome. ${ }^{4}$ The preserved relations, however, concern mainly elite circles - those of knyaz and senators. There were 'gentlemen' among the latter, they constituted only a small part of the community. A plenty of valuable information comes from letters, that may be found in preserved collections ${ }^{5}$ as well as in thematic editions from the pre-Revolutionary era. ${ }^{6}$ When analysing denominational issues, also court records (excerpts and regesta) may be

2 For a more detailed discussion, see H. Litwin, 'Fakcje magnackie na Kijowszczyźnie 1569-1648', in Wtadza i prestiż. Magnateria Rzeczypospolitej w XVI-XVIII wieku, ed. by J. Urwanowicz, Białystok, 2003.

3 J. Jerlicz, Letopisiec albo kroniczka, ed. by K.W. Wójcicki, vols. 1-2, Warsaw, 1853. 4 Litterae Episcoporum historiam Ucrainae illustrantes (1600-1900), ed. by A. Welykyj, vols. 1-2: 1600-1670, Rome, 1972-73: Litterae Nuntiorum Apostolicorum historiam Ucrainae illustrantes (1550-1850), ed. by A. Welykyj, vols. 1-6: 1550-1648, Rome, 1959-65: Epistolae Metropolitarum, ed. by A. Welykyj, vols. 1-2: 1613-1674, Rome, 1956.

5 See H. Litwin, 'Fakcje...', op. cit.

6 Archiv Jugozapadnoj Rossii izdavaemyj Vremennoju Komisseju dlja rozbora drevnich aktov, Part 1-8, vols. 1-34, Kiev, 1859-1914 (hereafter, AJZR with no. of Part/vol.); Archeografičeskij sbornik dokumentov otnosjaščichsja k istorii Severozapadnoj Rusi, vols. 1-7, Vilnius, 1867-70 (hereafter, Ar Sb with no. of vol.); Dokumenty objasnjajuščie istoriju Zapadno-Russkogo Kraja i ego otnošenÿa k Rossii i $k$ Polše, Petersburg, 1865; S.T. Golubev, Istorija Kievskoj Duchovnoj Akademii. Period do-Mogiljanskij, Priloženija, Kiev, 1880 (hereafter, IKDA); idem, Kievskij mitropolit Petr Mogita i ego spodvizniki, vols. 1-2: Priloženija, Kiev, 1883-98 (hereafter, Golubev - Mogila); Pamjatniki izdavaemye Kommisseju dlja rozbora drevnich aktov, vols. 1-4, Kiev, 1846-59 (hereafter, Pamjatniki, no. of vol. - no. of Part); Sbornik materialov dlja istoričeskoj topografii Kieva i ego okrestnostej, ed. by V. Antonovič, Kiev, 1874; Svodnaia galicko-russkaja letopis's 1600 po 1700 god, ed. by A.S. Petruševič, Lviv, 1874 (hereafter, Svodnaia); Dopolnenie do Svodnoj galicko-russkoj letopisi s 1600 po 1700 god, ed. by A.S. Petruševič, Lviv, 1891; Opis aktovoj knigi Kievskago Centralnago Archiva, vols. 1, 8-14, 16-18, Kiev, 1869-82 (hereafter, Opis); Ukraina (Kijów-Bractaw), Dział I-III, ed. by A. Jabłonowski, Warsaw, 1894 (Źródta Dziejowe, vols. 20-22); J.H. Drohojowski, Kronika Drohojowskich, vol. 2: Źródta, Cracow, 1904. 
included among the descriptive sources, that occasionally mention the denomination of the participants of legal tussles. ${ }^{7}$

A separate category of sources consists of individual public documents - foundation acts and testaments (or regesta of these). All such documents indicate the denomination of the issuers, and in certain cases also of the witnesses. Although donations to a 'foreign' religious institution (for example, an Orthodox temple being sponsored by a Catholic or a Protestant) cannot be excluded, no such case seems to be attested in the Kievan region.

Of the utmost importance for the present discussion are communal public documents, among them the numerous protestations and re-protestations concerning religious issues, as well as resolutions passed by gentry assemblies in religious matters, and especially the records of elections to high Orthodox offices. The extensive lists of signatories in those documents enable ascertaining the denomination of a significant part of the community under discussion. There may be some doubt in this case, especially about the denomination of the signatories of political and religious documents related to joint operations undertaken by dis-uniates and dissenters; these, however, can usually be resolved by referring to other sources. A list of the most important documents in this category is contained in Table 1.

Table 1. The most important sources related to the denomination of the representatives of Kievan gentry

\begin{tabular}{|l|l|l|l|}
\hline Document & Date & References & Abbreviation \\
\hline $\begin{array}{l}\text { An instruction for envoys sent to } \\
\text { the king by the Orthodox gentry } \\
\text { assembled at the synod in Brest }\end{array}$ & $\begin{array}{l}9 \text { October } \\
1596\end{array}$ & $\begin{array}{l}\text { AJZR 1-1, pp. } \\
515-16\end{array}$ & Instr. 1596 \\
\hline $\begin{array}{l}\text { A manifest of the Orthodox gentry in } \\
\text { defence of the brotherhood in Lublin }\end{array}$ & 5 May 1601 & $\begin{array}{l}\text { AJZR 2-1, pp. } \\
37-39\end{array}$ & Man. 1601 \\
\hline $\begin{array}{l}\text { A letter sent to the king by the } \\
\text { Orthodox gentry assembled in Lutsk }\end{array}$ & 1619 & $\begin{array}{l}\text { Pamjatniki 1-2, } \\
\text { p. } 12\end{array}$ & List 1619 \\
\hline $\begin{array}{l}\text { The document attesting Zachariasz } \\
\text { Kopysteński to have been elected as } \\
\text { the Pechersk archimandrite }\end{array}$ & $\begin{array}{l}28 \text { June } \\
1624\end{array}$ & $\begin{array}{l}\text { Golubev-Mogila, } \\
\text { pp. 271-72 }\end{array}$ & El. 1624 \\
\hline $\begin{array}{l}\text { The protest of the Orthodox gentry } \\
\text { against the synod in Kiev }\end{array}$ & 5 July 1629 & $\begin{array}{l}\text { Golubev-Mogila, } \\
\text { p. 366 }\end{array}$ & Prot. 1629 \\
\hline
\end{tabular}

7 The sources belonging to individual categories discussed in this paper are scattered throughout various collections and editions of manuscripts; relevant references in Table 2. 


\begin{tabular}{|c|c|c|c|}
\hline Document & Date & References & Abbreviation \\
\hline $\begin{array}{l}\text { The manifest of the Orthodox Kievan } \\
\text { gentry concerning the union of the } \\
\text { Bratsk and Pechersk schools }\end{array}$ & $\begin{array}{l}20 \\
\text { December } \\
1631\end{array}$ & IKDA, p. 71 & Man. 1631 \\
\hline $\begin{array}{l}\text { The document attesting Piotr Mohiła } \\
\text { to have been elected as the Kiev } \\
\text { metropolite }\end{array}$ & 1632 & $\begin{array}{l}\text { Kiev, Biblioteka } \\
\text { Ukrajinskoji } \\
\text { Akademiji Nauk } \\
\text { (hereafter, BUAN), } \\
\text { Ms. } 1441\end{array}$ & El. 1632 \\
\hline $\begin{array}{l}\text { The protest of the dissident Kievan } \\
\text { gentry concerning the course of the } \\
\text { sejmik }\end{array}$ & 1645 & AJZR 2-1, p. 281 & Prot. 1645 \\
\hline $\begin{array}{l}\text { The document attesting Jesyp Tryzna } \\
\text { to have been elected as the Pechersk } \\
\text { archimandrite }\end{array}$ & $\begin{array}{l}25 \text { January } \\
1647\end{array}$ & $\begin{array}{l}\text { AJZR 2-1, pp. } \\
341-42\end{array}$ & El. 1647 Tryzna \\
\hline $\begin{array}{l}\text { The document attesting Sylwester } \\
\text { Kossow to have been elected as the } \\
\text { Kiev metropolite }\end{array}$ & $\begin{array}{l}25 \text { January } \\
1647\end{array}$ & $\begin{array}{l}\text { AJZR 2-1, pp. } \\
347-48\end{array}$ & $\begin{array}{l}\text { El. } 1647 \\
\text { Kossow }\end{array}$ \\
\hline
\end{tabular}

Sources produced by religious institutions are only of a complementary significance. The borderlands gentry plays only a minor role in the published records of Protestant synods. ${ }^{8}$ An interesting source originating from the Orthodox church comprises pomianniki - lists of persons mentioned during commemorative services and masses. ${ }^{9}$ The usefulness of these documents is, however, limited as a result of the inconsistency in dating individual entries. Of Catholic origin are the chronicles of Jesuit posts (in Winnica, Fastów, Ksawerów) preserved in the congregation's archive in Rome. ${ }^{10}$ Unfortunately, these contain only scarce information related to the conversion of specific persons.

A significant - although still not a dominant - amount of information comes from the literature on the subject. An old study on the religious history of some Ruthenian noble families, written by P. Wiktorowski, still retains some academic value. ${ }^{11}$ Useful are also studies on the his-

8 Akta synodów różnowierczych w Polsce, vol. 3: Matopolska 1571-1632, ed. by M. Sipayłło, Warsaw, 1983.

9 Drevnij pomiannik Kievo-Michailovskogo (Zlatoverchnogo) Monastyrja (XVI-XVII w.), 'Čtenija v istoričeskom obščestve Nestora Letopisa', vols. 18-19, 1903, 1904.

10 Rome, Archivum Romanum Societatis Iesu (hereafter, ARSI), Polonia, no. 52, 66.

11 P.T. Viktorovskij, 'Zapadno-russkie dvorjanskie familii otapavšie ot' pravoslavija v końce XVI i v XVII u.', Trudy Kievskoj Duchovnoj Akademii', 1908, nos. 9-12; 1909, no. 6; 1910, nos. 3, 11; 1911, nos. 2, 6-8 (hereafter, Viktorovskij, year, no.). The work deals mainly with Ruthenian knyaz families. Concerning the families discussed in the present 
tory of Reformation on the Ruthenian lands of the Commonwealth. ${ }^{12}$ Moreover, works related to the Orthodox and Catholic pious foundations in Kiev region have been consulted, ${ }^{13}$ as well as monographs devoted to individual families and persons. Some of the persons listed in Table 1 have their biographical entries in Polski Stownik Biograficzny. ${ }^{14}$ Separate biographical studies have been so far written only for Adam Kisiel and Jerzy Niemirycz, ${ }^{15}$ although the works differ significantly in their quality. The histories of some Kievan noble families were studied by historians and essayists in the nineteenth century; although the methodology of those works is outdated, their authors had access to sources that have been lost since that time. ${ }^{16}$

paper, Wiktorowski studied the histories of Siemaszko (1911, no. 7/8), Tyszkiewicz, Skumin, and Łohojski families (1911, no. 2), as well as Niemirycz (1911, no. 7/8).

12 A. Kossowski, 'Zarys dziejów protestantyzmu na Wołyniu w XVI-XVII w.', Rocznik Wotyński, 3, 1934; O. Levickij, 'Socinjanstvo v Polše i jugozapadnoj Rusi', Kievskaja Starina, 1, 1882, 4, 5, 6 (an abridged Polish version of this work has been published in Reformacja w Polsce, 2, 1922); J. Tazbir, 'Antytrynitaryzm na ziemiach ukraińskich w XVII w.', in $Z$ polskich studiów slawistycznych, Series 4: Historia, no. 3, Warsaw, 1972; idem, 'Kisielińsko-bereski zbór Braci Polskich', Przeglad Historyczny, 57, 1966; H. Merczyng, Zbory i senatorowie protestanccy $w$ dawnej Rzeczypospolitej, Warsaw, 1905, p. 128; H. Lulewicz, 'Skład wyznaniowy senatorów świeckich Wielkiego Księstwa Litewskiego za panowania Wazów', Przegląd Historyczny, 68, 1977, 3, p. 435. 13 Wołyniak [J.M. Giżycki], Wykaz klasztorów dominikańskich prowincji ruskiej, Cracow, 1923, p. 213; Wołyniak [J.M. Giżycki], 'Zniesione kościoły i klasztory katolickie przez rząd rosyjski w wieku XIX w diecezji łuckiej, żytomierskiej i kamienieckiej’, Nova Polonia Sacra, 1, 1928; S.T. Golubev, 'K biografii fundatorši Kievo-bratskogo monastyrja Elizavety Vasylievny Hulevičovny i eja bližajšych rodstvennikov', Trudy Kievskoj Duchovnoj Akademii, 1886; Klasztory Bernardyńskie w Polsce w jej granicach historycznych, Kalwaria Zebrzydowska, 1985, p. 215; H. Litwin, 'Dobra ziemskie Cerkwi Prawosławnej i Kościoła Katolickiego obu obrządków na Kijowszczyźnie w świetle akt skarbowych i sądowych 1569-1648', Rocznik Teologiczny, 32, 1990, 2; M.A. Maksimovicz, Sobranie sočinenii, vols. 1-3, Kiev, 1876-1900; B.J. Wanat, Zakon Karmelitów Bosych w Polsce. Klasztory karmelitów i karmelitanek bosych 1605-1977, Cracow, 1979. 14 Andrzej Chalecki, Iwan and Jerzy Czaplica-Szpanowski, Hawryło and Roman Hojski (in PSB as Hosccy), Gabriel Hornostaj, Adam Kisiel, Filon Kmita-Czarnobylski, Stefan, Jerzy and Władysław Niemirycz, Mikołaj and Aleksander Siemaszko. The entries of Chalecki and Hornostaj do not contain any information about their faith.

15 F. Sysyn, Between Poland and the Ukraine. The Dilemma of Adam Kysil 1600-1653, Cambridge, 1985; S. Kot, Jerzy Niemirycz. W 300-lecie Ugody Hadziackiej, Paris, 1960; M. Brik, Jurij Nemyryč na tli istoriji Ukrajiny, Losser, 1974.

16 Short monographs on individual families: O. Halecki, 'Chaleccy na Ukrainie', Miesięcznik Heraldyczny, 3, 1910; A. Kamieński, 'Kariera rodu Siemaszków w XV-XVII 
The denomination of members of the Kievan noble families

The community under discussion includes members of the noble families that were active in the Kiev region in 1569-1648. The list has been based on data collected by the present author elsewhere. ${ }^{17}$ All adult males are taken into consideration, who acted independently as landowners. Omitted are those members of the families who did not own any estates or held offices in the Kiev region in the period under discussion. This way, 260 persons have been selected, and it was the author's aim, to ascertain their faith. All relevant information is presented in Table 2, containing data related to the denomination of particular members of the noble families. The earlier collected data and published results of research contain no evidence that any of nobles converted to Roman Catholicism in the Ruthenian lands of the Commonwealth has ever returned to the Orthodox faith. There is no evidence that any of their descendants converted to the Orthodoxy either ${ }^{18}$. Therefore I assumed that the lineal ancestors of persons described as being of 'the Greek religion' must have been of the same denomination.

wieku', Lituano-Slavica Posnaniensia. Studia Historica, 3, 1989; Z. Lasocki, 'Sienkiewiczowskie "lwie pacholę" i ród jego', Miesięcznik Heraldyczny, 15, 1936 (on the Aksak family); A.J. Rolle, 'Niemierycze', in idem, Opowiadania historyczne, Series 3, Warsaw, 1882; T.J. Stecki, 'Steccy herbu Radwan', in T. Żychliński, Ztota księga szlachty polskiej, vols. 1-31, Poznań, 1879-1908, vol. 10, pp. 3-13; E. Tyszkiewicz, Groby rodziny Tyszkiewiczów, Warsaw, 1873. E. Rulikowski (in his works: Opis powiatu kijowskiego, Kiev and Warsaw, 1913, and Opis powiatu wasylkowskiego pod wzgledem historycznym, obyczajowym i statystycznym, Warsaw, 1853) describes, among others, the stories of Aksak, Hornostaj, Iwaszeńcewicz-Makarewicz, and Sołtan families. T.J. Stecki $(Z$ boru $i$ stepu, Cracow, 1888) deals with Babiński and Czaplica families. A. Jabłonowski and E. Rulikowski wrote a series of papers on specific estates and their owners; those have been published in Stownik Geograficzny Królestwa Polskiego i krajów ościennych, vols. 1-13, Warsaw, 1880-93 (hereafter, SGKP and a specific entry). See also, A.J. Rolle, 'Dzieje szlachty okolicznej w owruckim powiecie', in idem, $Z$ przesztości Polesia Kijowskiego. Opowiadania historyczne, Warsaw, 1882, p. 22.

17 H. Litwin, 'Rody pańskie Kijowszczyzny 1569-1648. Status majątkowy', Przegląd Wschodni, 8, 2002, 2 (30), pp. 235-92.

18 Idem, 'Katolicyzacja szlachty ruskiej i procesy asymilacyjne na Ukrainie 1569-1648', in Triumfy i porażki. Studia z dziejów kultury polskiej XVI-XVIII w., ed. by M. Bogucka, Warsaw, 1989, pp. 56-66. 
Table 2. The denomination of members of the Kievan noble families, 1569-1648 ${ }^{19}$

\begin{tabular}{|c|c|c|c|c|}
\hline Name & 1 & 2 & 3 & 4 \\
\hline \multicolumn{5}{|l|}{ Aksak } \\
\hline Martyn $\dagger$ after 1569 & $\mathrm{O}$ & & & \\
\hline $\begin{array}{l}\text { Jan (Iwan) Martynowicz } \\
\dagger 1627\end{array}$ & $\mathrm{O}$ & $\begin{array}{l}1624 \text {, before } \\
1627\end{array}$ & $\begin{array}{l}\text { document, } \\
\text { report }\end{array}$ & $\begin{array}{l}\text { El. } 1624 ; \text { Z. Lasocki, op. } \\
\text { cit., p. } 4\end{array}$ \\
\hline Stefan Iwanowicz- & $\mathrm{O}$ & before 1627 & report & $\begin{array}{l}\text { Golubev - Mogiła, p. } \\
293\end{array}$ \\
\hline--- & $\mathrm{C}$ & 1627 & report & ibid. \\
\hline Michał Iwanowicz- & $?$ & & & \\
\hline \multicolumn{5}{|l|}{ Babiński } \\
\hline Andrzej † before 1598 & $\mathrm{O}$ & 1595 & document & Pamiatniki 3-1, p. 91 \\
\hline $\begin{array}{l}\text { Wasyl Andrzejowicz } \dagger \\
\text { before } 1624\end{array}$ & $\mathrm{O}$ & 1597,1601 & $\begin{array}{l}\text { document, } \\
\text { report }\end{array}$ & $\begin{array}{l}\text { Ar. Sb. 1, p. 229; } \\
\text { Diariusze sejmowe r. } \\
\text { 1597, ed. by } \\
\text { E. Barwiński, Cracow, } \\
1907 \text {, p. } 400\end{array}$ \\
\hline--- & A & before $\dagger$ & literature & $\begin{array}{l}\text { A. Kossowski, op. cit., } \\
\text { p. } 245\end{array}$ \\
\hline Piotr Wasylowicz- & A & & literature & $\begin{array}{l}\text { A. Kossowski, op. cit., } \\
\text { p. } 245\end{array}$ \\
\hline Eliasz Wasylowicz- & A & 1644 & literature & $\begin{array}{l}\text { T.J. Stecki, op. cit., p. } \\
121\end{array}$ \\
\hline \multicolumn{5}{|l|}{ Butowicz } \\
\hline Jacek Dymitrowicz $† 1604$ & $\mathrm{O}$ & 1596 & document & Instr. 1596 \\
\hline Hryhory Jackowicz $† 1591$ & $\mathrm{O}$ & & & \\
\hline Semen Jackowicz $† 1601$ & $\mathrm{O}$ & 1601 & document & $\begin{array}{l}\text { AJZR 1-4, p. } 12 \text {; Opis, } \\
\text { vol. } 19 \text {, p. } 28\end{array}$ \\
\hline $\begin{array}{l}\text { Aleksander Jackowicz } \dagger \\
1604 / 05\end{array}$ & $\mathrm{O}$ & & & \\
\hline Marcin Jackowicz † 1630 & $\mathrm{O}$ & $\begin{array}{l}1596,1601 \\
1629\end{array}$ & document & $\begin{array}{l}\text { Instr. 1596; Man. 1601; } \\
\text { Golubev - Mogiła, p. } \\
366\end{array}$ \\
\hline Paweł Marcinowicz- & $\mathrm{O}$ & 1648 & report & $\begin{array}{l}\text { J. Jerlicz, op. cit., vol. } 1 \text {, } \\
\text { p. } 168\end{array}$ \\
\hline Chalecki & & & & \\
\hline
\end{tabular}

19 Column 1 indicates the denomination, where A - Arian, P - Protestant-Calvinist, C - Catholic, O - Orthodox, U - Uniate. Column 2 contains dates of the information related to the person's denomination; column 3 - type of source, and column 4 - references. 


\begin{tabular}{|c|c|c|c|c|}
\hline Name & 1 & 2 & 3 & 4 \\
\hline $\begin{array}{l}\text { Wasyl Harasymowicz until } \\
1575\end{array}$ & $?$ & & & \\
\hline $\begin{array}{l}\text { Bohdan Harasymowicz } \\
\text { until } 1575\end{array}$ & $?$ & & & \\
\hline $\begin{array}{l}\text { Andrzej Jesypowicz } \dagger \\
\text { before } 1595\end{array}$ & $?$ & & & \\
\hline Jan Jesypowicz $\dagger 1610$ & ? & & & \\
\hline $\begin{array}{l}\text { Wasyl Jesypowicz } \dagger \text { ca. } \\
1588\end{array}$ & $?$ & & & \\
\hline $\begin{array}{l}\text { Józefian Andrzejowicz } \\
\text { before } 1625\end{array}$ & ? & & & \\
\hline \multicolumn{5}{|l|}{ Chreptowicz-Bohuryński } \\
\hline $\begin{array}{l}\text { Konstanty } 1618 \dagger \text { after } \\
1645\end{array}$ & A & 1645 & document & Prot. 1645 \\
\hline $\begin{array}{l}\text { Władysław } \\
\text { Konstantynowicz- }\end{array}$ & A & 1645 & document & Prot. 1645 \\
\hline \multicolumn{5}{|l|}{ Czaplica-Szpanowski } \\
\hline Iwan $1585 \dagger 1607$ & $\mathrm{P}$ & 1607 & literature & $P S B$, vol. 4, p. 170 \\
\hline Jerzy 1639 & A & 1648 & literature & $P S B$, vol. 4, p. 170 \\
\hline \multicolumn{5}{|l|}{ Dorohostalski } \\
\hline $\begin{array}{l}\text { Piotr Mikołajowicz before } \\
1588\end{array}$ & $?$ & & & \\
\hline Paweł Janowicz $† 1607 / 9$ & ? & & & \\
\hline \multicolumn{5}{|l|}{ Furs } \\
\hline Iwan until after 1581 & $\mathrm{O}$ & & document & Ar. Sb. 6, p. 324 \\
\hline \multicolumn{5}{|l|}{ Hojski } \\
\hline Hawryło $† 1632$ & $\mathrm{O}$ & 1596,1601 & document & Instr. 1596; Man. 1601 \\
\hline--- & A & before 1610 & literature & $P S B$, vol. 10 , p. 25 \\
\hline $\begin{array}{l}\text { Roman Hawryłowicz } \dagger \\
1635\end{array}$ & $\mathrm{O}$ & before 1617 & literature & $P S B$, vol. 10 , p. 26 \\
\hline--- & A & 1617 & literature & $P S B$, vol. 10 , p. 26 \\
\hline \multicolumn{5}{|l|}{ Hornostaj } \\
\hline Hawryło (Gabriel) † 1587 & $\mathrm{O}$ & & & \\
\hline--- & $\mathrm{P}$ & & literature & $\begin{array}{l}\text { H. Merczynfi, op. cit., } \\
\text { p. } 128\end{array}$ \\
\hline $\begin{array}{l}\text { Jerofiej (Hieronim) } \\
\text { Hawryłowicz } † 1600\end{array}$ & $\mathrm{P}$ & & & \\
\hline
\end{tabular}




\begin{tabular}{|c|c|c|c|c|}
\hline Name & 1 & 2 & 3 & 4 \\
\hline $\begin{array}{l}\text { Samuel Jerofiejowicz } \dagger \\
1618\end{array}$ & $\mathrm{P}$ & 1605,1618 & document & $\begin{array}{l}\text { Synody..., op. cit., p. } \\
\text { 272; E. Rulikowski, } \\
\text { Opis powiatu } \\
\text { kijowskiego..., p. } 129\end{array}$ \\
\hline $\begin{array}{l}\text { Michał Samuelowicz } \dagger \\
1637\end{array}$ & $\mathrm{P}$ & 1637 & literature & E. Rulikowski, op. cit. \\
\hline \multicolumn{5}{|l|}{ Hulewicz-Dolski } \\
\hline Mikołaj 1587-† 1606 & $\mathrm{O}$ & & document & AJZR 1-6, p. 678 \\
\hline \multicolumn{5}{|l|}{ Hulewicz-Wojutyniec } \\
\hline Łukasz 1646- & $\mathrm{P}$ & 1649 & literature & $\begin{array}{l}\text { S. Ochmann, Sejm } \\
\text { koronacyjny w } 1649 \text { r., } \\
\text { Wrocław, 1985, p. } 225\end{array}$ \\
\hline \multicolumn{5}{|l|}{ Iwaszeńcewicz-Makarewicz } \\
\hline Iwan $\dagger$ after 1591 & $\mathrm{O}$ & & & \\
\hline Hryhory $\dagger$ after 1591 & $\mathrm{O}$ & & & \\
\hline Mikołaj † 1621 & $\mathrm{O}$ & 1591 & literature & $\begin{array}{l}\text { E. Rulikowski, op. cit., } \\
\text { p. } 155\end{array}$ \\
\hline--- & $\mathrm{P}$ & 1621 & literature & ibid. \\
\hline $\begin{array}{l}\text { Krzysztof Mikołajowicz } \\
\dagger 1641\end{array}$ & $\mathrm{P}$ & 1641 & literature & ibid. \\
\hline $\begin{array}{l}\text { Aleksander Mikołajowicz } \\
\dagger 1613\end{array}$ & $\mathrm{P}$ & 1613 & literature & ibid. \\
\hline $\begin{array}{l}\text { Andrzej Mikołajowicz } \dagger \\
1618\end{array}$ & $\mathrm{P}$ & 1618 & literature & ibid. \\
\hline Jerzy Mikołajowicz $† 1632$ & $\mathrm{P}$ & before 1620 & literature & ibid. \\
\hline--- & $\mathrm{C}$ & 1620,1632 & literature & ibid. \\
\hline \multicolumn{5}{|l|}{ Jelec } \\
\hline Dymitr $\dagger 1598$ & $\mathrm{O}$ & 1598 & literature & $\begin{array}{l}\text { M.A. Maksimovicz, op. } \\
\text { cit., vol. 2, p. } 234\end{array}$ \\
\hline Filon Dymitrowicz $\dagger 1625$ & $\mathrm{O}$ & $\begin{array}{l}1608,1624 \\
1625\end{array}$ & report & $\begin{array}{l}\text { ARSI, Pol. 75, no. } \\
\text { 18; BUAN, Ms. } \\
\text { 23371; El. 1624; } \\
\text { M.A. Maksimovicz, op. } \\
\text { cit., vol. 2, p. } 234\end{array}$ \\
\hline $\begin{array}{l}\text { Konstanty Dymitrowicz } \\
\dagger 1634\end{array}$ & $\mathrm{O}$ & 1608 & document & BUAN, Ms. 23371 \\
\hline Fedor Dymitrowicz $\dagger 1648$ & $\mathrm{O}$ & 1624 & $\begin{array}{l}\text { document, } \\
\text { literature }\end{array}$ & $\begin{array}{l}\text { El. 1624; A.J. Rolle, } \\
\text { Dzieje szlachty..., op. } \\
\text { cit., p. } 22\end{array}$ \\
\hline
\end{tabular}




\begin{tabular}{|c|c|c|c|c|}
\hline Name & 1 & 2 & 3 & 4 \\
\hline $\begin{array}{l}\text { Aleksander (Ignacy) } \\
\text { Filonowicz- }\end{array}$ & $\mathrm{O}$ & before 1632 & report & $\begin{array}{l}\text { ARSI Pol. } 74 \text {, no. } \\
\text { 139/397, 76, no. } 18\end{array}$ \\
\hline--- & C & 1632,1646 & document & $\begin{array}{l}\text { ARSI Pol. } 74 \text {, no. } \\
\text { 139/397, } 76 \text {, no. } 18 \text {; } \\
\text { Opis, vol. } 17, \text { p. } 47\end{array}$ \\
\hline Remigian Fedorowicz- & $\mathrm{O}$ & before 1647 & & \\
\hline--- & $\mathrm{C}$ & 1647 & report & ARSI Pol. 77, 1. 371 \\
\hline \multicolumn{5}{|l|}{ Jełowicki } \\
\hline Zachariasz $1613-\dagger 1629$ & $\mathrm{O}$ & before 1596 & & \\
\hline--- & $\mathrm{U}$ & 1598 & document & AJZR 1-10, p. 501 \\
\hline--- & $\mathrm{O}$ & 1624 & document & El. 1624 \\
\hline Aleksander 1643- & $\mathrm{O}$ & 1646 & report & $\begin{array}{l}\text { Cracow, Czartoryski } \\
\text { Library (hereafter, } \\
\text { BC), Ms. 1657, 1. 345; } \\
\text { Golubev - Mogila p. } \\
338\end{array}$ \\
\hline \multicolumn{5}{|l|}{ Kisiel } \\
\hline Adam since $1628-$ & $\mathrm{O}$ & & literature & $\begin{array}{l}\text { F. Sysyn, Between..., op. } \\
\text { cit., p. } 69\end{array}$ \\
\hline \multicolumn{5}{|l|}{ Kmita-Czarnobylski } \\
\hline Filon $\dagger 1585$ & $\mathrm{O}$ & 1585 & literature & $P S B$, vol. 13 , pp. $88-89$ \\
\hline Lazar Filonowicz $† 1595$ & $\mathrm{O}$ & 1595 & literature & $P S B$, vol. 13, pp. $88-89$ \\
\hline \multicolumn{5}{|l|}{ Kordysz } \\
\hline Michał until 1588 & $\mathrm{O}$ & & document & $\begin{array}{l}\text { Cracow, State Archives } \\
\text { of the Voivodeship } \\
\text { (hereafter, WAPK), } \\
\text { Archiwum Sanguszków, } \\
\text { Teki Rzymskie, no. } \\
\text { XVIIa, b. p. }\end{array}$ \\
\hline \multicolumn{5}{|l|}{ Kotaszewicz } \\
\hline Wasyl until 1595 & ? & & & \\
\hline \multicolumn{5}{|l|}{ Łasko } \\
\hline Iwan Olechnowicz $\dagger 1581$ & $\mathrm{O}$ & & & \\
\hline $\begin{array}{l}\text { Michał Olechnowicz } \dagger \\
\text { after } 1607\end{array}$ & $\mathrm{O}$ & & & \\
\hline Fedor Olechnowicz $\dagger 1592$ & $\mathrm{O}$ & & & \\
\hline $\begin{array}{l}\text { Kuźma Iwanowicz } \dagger \text { after } \\
1618\end{array}$ & $?$ & & & \\
\hline
\end{tabular}




\begin{tabular}{|c|c|c|c|c|}
\hline Name & 1 & 2 & 3 & 4 \\
\hline $\begin{array}{l}\text { Wasyl Fedorowicz } \dagger \text { after } \\
1615\end{array}$ & ? & & & \\
\hline $\begin{array}{l}\text { Andrzej Fedorowicz } \dagger \text { after } \\
1629\end{array}$ & $?$ & & & \\
\hline $\begin{array}{l}\text { Michał Michałowicz } \dagger \\
1633\end{array}$ & $\mathrm{O}$ & 1632 & $\begin{array}{l}\text { document, } \\
\text { report }\end{array}$ & $\begin{array}{l}\text { El. 1632; Drevnij } \\
\text { pomiannik..., op. cit., } \\
\text { p. } 55\end{array}$ \\
\hline Iwan Andrzejowicz - & $?$ & & & \\
\hline Fedor Michałowicz - & $\mathrm{O}$ & & report & $\begin{array}{l}\text { Drevnij pomiannik..., } \\
\text { op. cit., p. } 55\end{array}$ \\
\hline \multicolumn{5}{|l|}{ Łodziat } \\
\hline Stefan $1610-29$ & $?$ & & & \\
\hline \multicolumn{5}{|l|}{ Łozka } \\
\hline $\begin{array}{l}\text { Filon Jackowicz } \dagger \text { before } \\
1578\end{array}$ & $\mathrm{O}$ & & & \\
\hline $\begin{array}{l}\text { Wasyl Jackowicz } \dagger \text { before } \\
1581\end{array}$ & $\mathrm{O}$ & & & \\
\hline Stefan Wasylowicz $\dagger 1618$ & $\mathrm{O}$ & 1599,1615 & document & $\begin{array}{l}\text { Dokumjenty } \\
\text { objasnjajuščija..., p. } \\
\text { 206; IKDA, p. } 9\end{array}$ \\
\hline Iwan Wasylowicz † 1608 & $\mathrm{O}$ & & & \\
\hline Ławryn Wasylowicz $† 1618$ & $\mathrm{O}$ & 1618 & document & AJZR 1-6, p. 678 \\
\hline Józef Stefanowicz † 1631 & $\mathrm{O}$ & 1624 & document & El. 1624 \\
\hline Michał Stefanowicz $\dagger 1648$ & $\mathrm{O}$ & & & \\
\hline--- & $\mathrm{C}$ & 1648 & document & $\begin{array}{l}\text { S.T. Golubev, K } \\
\text { biografii..., op. cit., p. } 39\end{array}$ \\
\hline \multicolumn{5}{|l|}{ Niemirycz } \\
\hline Jesyp $† 1598$ & $\mathrm{O}$ & 1598 & report & $\begin{array}{l}\text { Ukraina..., op. cit., Dz. } \\
\text { 2, p. } 373\end{array}$ \\
\hline Semen Jesypowicz $\dagger 1605$ & $\mathrm{O}$ & & & \\
\hline Matwij Jesypowicz $† 1613$ & $\mathrm{O}$ & 1601 & document & Man. 1601 \\
\hline $\begin{array}{l}\text { Iwan Jesypowicz } \dagger \text { after } \\
1618\end{array}$ & $\mathrm{O}$ & & & \\
\hline Andrzej Jesypowicz $† 1607$ & $\mathrm{O}$ & 1607 & literature & $P S B$, vol. 22 , p. 819 \\
\hline Iwan Iwanowicz $\dagger ?$ & $\mathrm{O}$ & & literature & $\begin{array}{l}\text { A.J. Rolle, } \\
\text { Niemierycze..., op. cit., } \\
\text { p. } 22\end{array}$ \\
\hline
\end{tabular}


DENOMINATIONAL COMPOSITION OF THE KIEVAN...

\begin{tabular}{|c|c|c|c|c|}
\hline Name & 1 & 2 & 3 & 4 \\
\hline $\begin{array}{l}\text { Michał Iwanowicz } \dagger \text { after } \\
1628\end{array}$ & $\mathrm{O}$ & & literature & ibid. \\
\hline $\begin{array}{l}\text { Krzysztof Matwijowicz } \dagger \\
1618\end{array}$ & $\mathrm{O}$ & & literature & ibid. \\
\hline $\begin{array}{l}\text { Mikołaj Matwijowicz } \dagger \\
\text { after } 1620\end{array}$ & $\mathrm{O}$ & & literature & ibid. \\
\hline $\begin{array}{l}\text { Semen Matwijowicz } \dagger \\
\text { after } 1620\end{array}$ & $\mathrm{O}$ & & literature & ibid. \\
\hline $\begin{array}{l}\text { Aleksander Matwijowicz } \\
\dagger 1648\end{array}$ & $\mathrm{O}$ & $\begin{array}{l}1619,1624 \\
1629,1648\end{array}$ & document & $\begin{array}{l}\text { List 1619; El. 1624; } \\
\text { Prot. 1629; A.J. Rolle, } \\
\text { Niemierycze..., op. cit., } \\
\text { p. } 22\end{array}$ \\
\hline $\begin{array}{l}\text { Stefan Andrzejowicz } \dagger \\
1630\end{array}$ & $\mathrm{O}$ & & literature & PSB, vol. 22. p. 819 \\
\hline--- & A & 1630 & literature & PSB, vol. 22 , p. 819 \\
\hline Jerzy Stefanowicz - & A & 1648 & literature & $P S B$, vol. 22 , pp. $811-13$ \\
\hline Wladysław Stefanowicz - & A & 1648 & literature & $P S B$, vol. 22, p. 824 \\
\hline \multicolumn{5}{|l|}{ Olizar-Wołczkowicz } \\
\hline Iwan $† 1577$ & $\mathrm{O}$ & & & \\
\hline Adam Iwanowicz $† 1625$ & $\mathrm{O}$ & before 1602 & literature & SGKP 'Korosteszów’ \\
\hline--- & C & 1602 & literature & SGKP 'Korosteszów’ \\
\hline $\begin{array}{l}\text { Wojciech Iwanowicz } \dagger \\
1628\end{array}$ & $\mathrm{C}$ & & & \\
\hline $\begin{array}{l}\text { Ludwik Adamowicz } \dagger \\
1645\end{array}$ & $\mathrm{C}$ & 1645 & literature & SGKP 'Korosteszów’ \\
\hline Michal - & $\mathrm{C}$ & & & \\
\hline Jan - & C & & & \\
\hline Stanisław - & $\mathrm{C}$ & & & \\
\hline \multicolumn{5}{|l|}{ Pawsza } \\
\hline Bohufał $\uparrow 1598$ & $\mathrm{O}$ & 1569,1595 & $\begin{array}{l}\text { document, } \\
\text { report }\end{array}$ & $\begin{array}{l}\text { Cracow, Library of } \\
\text { the Polish Academy } \\
\text { of Sciences (hereafter, } \\
\text { BPANK), Ms. 2983, } 1 . \\
\text { 4; Jagiellonian Library } \\
\text { (hereafter, BJ), Ms. } \\
6049 \text {, p. } 73\end{array}$ \\
\hline $\begin{array}{l}\text { Jakub Bohufałowicz } \dagger \\
\text { before } 1613\end{array}$ & $\mathrm{O}$ & & & \\
\hline
\end{tabular}




\begin{tabular}{|c|c|c|c|c|}
\hline Name & 1 & 2 & 3 & 4 \\
\hline $\begin{array}{l}\text { Fedor Bohufałowicz } \dagger \\
1624\end{array}$ & $\mathrm{O}$ & & & \\
\hline $\begin{array}{l}\text { Iwan Jakubowicz } \dagger \text { ca. } \\
1641\end{array}$ & $?$ & & & \\
\hline $\begin{array}{l}\text { Wasyl Jakubowicz } \dagger \text { ca. } \\
1641\end{array}$ & $?$ & & & \\
\hline Aleksander Jakubowicz & $?$ & & & \\
\hline Szymon Fedorowicz - & $\mathrm{O}$ & 1631,1647 & document & $\begin{array}{l}\text { Man. 1631; El. } 1647 \\
\text { Tryzna }\end{array}$ \\
\hline \multicolumn{5}{|l|}{ Prezowski } \\
\hline Semen $†$ ca. 1587 & ? & & & \\
\hline Iwan Semenowicz $\dagger 1606$ & ? & & & \\
\hline Iwan Iwanowicz $\dagger 1643$ & $?$ & & & \\
\hline $\begin{array}{l}\text { Semen Iwanowicz } \dagger \mathrm{ca} . \\
1643\end{array}$ & ? & & & \\
\hline Mikołaj Iwanowicz - & $?$ & & & \\
\hline \multicolumn{5}{|l|}{ Proskur-Suszczański } \\
\hline $\begin{array}{l}\text { Iwan Tymofiejowicz } \dagger \\
1599\end{array}$ & $\mathrm{O}$ & & & \\
\hline $\begin{array}{l}\text { Bohdan Tymofiejowicz } \dagger \\
\text { before } 1581\end{array}$ & $\mathrm{O}$ & & & \\
\hline $\begin{array}{l}\text { Hryhory Bohdanowicz } \dagger \\
1608\end{array}$ & $\mathrm{O}$ & 1608 & document & BUAN, Ms. 23371 \\
\hline $\begin{array}{l}\text { Michał Bohdanowicz } \dagger \\
\text { after } 1602\end{array}$ & ? & & & \\
\hline $\begin{array}{l}\text { Jerzy Michałowicz } \dagger \text { after } \\
1611\end{array}$ & ? & & & \\
\hline $\begin{array}{l}\text { Michał Michałowicz } \dagger \text { after } \\
1646\end{array}$ & $?$ & & & \\
\hline Fedor Hryhorowicz $\dagger 1647$ & $\mathrm{O}$ & $\begin{array}{l}1624,1631 \\
1647\end{array}$ & document & $\begin{array}{l}\text { El. 1624; Man. 1631; El. } \\
1647 \text { Kossow }\end{array}$ \\
\hline $\begin{array}{l}\text { Mikołaj Hryhorowicz } \dagger \\
1616\end{array}$ & $\mathrm{O}$ & & & \\
\hline Jerzy Hryhorowicz - & $\mathrm{O}$ & & & \\
\hline Piotr Fedorowicz - & $\mathrm{O}$ & 1632 & & \\
\hline Jan Fedorowicz - & $\mathrm{O}$ & 1648 & document & AJZR 2-2, p. 387 \\
\hline Siemaszko & & & & \\
\hline
\end{tabular}




\begin{tabular}{|c|c|c|c|c|}
\hline Name & 1 & 2 & 3 & 4 \\
\hline Aleksander $\dagger 1587$ & $\mathrm{O}$ & & literature & $\begin{array}{l}\text { A. Kamieński, Kariera } \\
\text { rodu Siemaszków..., op. } \\
\text { cit., p. } 194\end{array}$ \\
\hline--- & C & 1586 & literature & op. cit. \\
\hline $\begin{array}{l}\text { Mikołaj Aleksandrowicz } \\
\dagger 1617\end{array}$ & C & 1586 & literature & op. cit., p. 200 \\
\hline \multicolumn{5}{|l|}{ Służka } \\
\hline Mikołaj $\dagger 1598 / 1602$ & $\mathrm{P}$ & & & \\
\hline $\begin{array}{l}\text { Krzysztof Mikołajowicz } \\
\dagger 1620\end{array}$ & $?$ & & & \\
\hline $\begin{array}{l}\text { Aleksander Mikołajowicz } \\
\text { until before } 1640\end{array}$ & $\mathrm{P}$ & & literature & $\begin{array}{l}\text { J.M. Giżycki } \\
\text { (Wołyniak), Wykaz } \\
\text { klasztorów..., op. cit., } \\
\text { p. 213; H. Lulewicz, } \\
\text { Skład.... op. cit., p. } 435\end{array}$ \\
\hline--- & C & 1620,1634 & literature & J.M. Giżycki, op. cit. \\
\hline $\begin{array}{l}\text { Remigian Krzysztofowicz } \\
\text { until } 1640\end{array}$ & $?$ & & & \\
\hline \multicolumn{5}{|l|}{ Sołtan } \\
\hline Fedor $\dagger 1576$ & $\mathrm{O}$ & & & \\
\hline $\begin{array}{l}\text { Piotr Fedorowicz } \dagger \text { before } \\
1598\end{array}$ & $\mathrm{O}$ & & & \\
\hline Iwan Fedorowicz $\dagger 1613$ & $\mathrm{O}$ & & & \\
\hline $\begin{array}{l}\text { Paweł Fedorowicz } \dagger \text { after } \\
1602\end{array}$ & $\mathrm{O}$ & & & \\
\hline $\begin{array}{l}\text { Jurij Piotrowicz } \dagger \text { after } \\
1602\end{array}$ & $?$ & & & \\
\hline $\begin{array}{l}\text { Bohdan Pawłowicz } \dagger \text { after } \\
1634\end{array}$ & $\mathrm{O}$ & 1631 & document & Man. 1631 \\
\hline $\begin{array}{l}\text { Stefan Pawłowicz } \dagger \text { after } \\
1628\end{array}$ & $?$ & & & \\
\hline Mikołaj Bohdanowicz - & $\mathrm{O}$ & 1633 & literature & $\begin{array}{l}\text { J. Dzięgielewski, } \\
\text { O tolerancje dla } \\
\text { zdominowanych. } \\
\text { Polityka wyznaniowa } \\
\text { Rzeczypospolitej w latach } \\
\text { panowania Wtadystawa } \\
\text { IV, Warsaw, 1986, p. } 64\end{array}$ \\
\hline Samuel Bohdanowicz - & $?$ & & & \\
\hline
\end{tabular}




\begin{tabular}{|c|c|c|c|c|}
\hline Name & 1 & 2 & 3 & 4 \\
\hline $\begin{array}{l}\text { Kiryk Iwanowicz } \dagger \mathrm{ca} \text {. } \\
1624\end{array}$ & $\mathrm{O}$ & & & \\
\hline Iwan Kirykowicz - & $?$ & & & \\
\hline Fedor Kirykowicz - & $\mathrm{O}$ & 1647 & document & El. 1647 Kossow \\
\hline \multicolumn{5}{|l|}{ Aleksander - } \\
\hline \multicolumn{5}{|l|}{ Stecki } \\
\hline Stefan Olechnowicz $\dagger 1593$ & $\mathrm{O}$ & 1593 & literature & T.J. Stecki, op. cit., p. 3 \\
\hline $\begin{array}{l}\text { Mikołaj Stefanowicz } \dagger \\
1627\end{array}$ & $\mathrm{O}$ & 1615 & document & IKDA, p. 9 \\
\hline $\begin{array}{l}\text { Krzysztof Stefanowicz } \dagger \\
1626\end{array}$ & $\mathrm{O}$ & 1626 & literature & T.J. Stecki, op. cit., p. 3 \\
\hline $\begin{array}{l}\text { Paweł Stefanowicz } \dagger \text { after } \\
1641\end{array}$ & ? & & & \\
\hline $\begin{array}{l}\text { Fedor Stefanowicz } \dagger \text { after } \\
1629\end{array}$ & $\mathrm{O}$ & 1629 & document & Prot. 1629 \\
\hline Aleksander Mikołajowicz - & $\mathrm{O}$ & 1648 & report & Svodnaia, p. 440 \\
\hline Jan Mikołajowicz - & $?$ & & & \\
\hline Samuel Mikołajowicz - & $?$ & & & \\
\hline \multicolumn{5}{|l|}{ Strybyl } \\
\hline Bohdan Iwanowicz $† 1605$ & $\mathrm{O}$ & & & \\
\hline Filon Bohdanowicz $\dagger 1634$ & $\mathrm{O}$ & 1624,1631 & document & El. 1624; Man. 1631 \\
\hline $\begin{array}{l}\text { Fedor Bohdanowicz } \dagger \\
1609\end{array}$ & $\mathrm{O}$ & & & \\
\hline $\begin{array}{l}\text { Daniło (Daniel) } \\
\text { Bohdanowicz } \dagger \text { after } 1630\end{array}$ & $\mathrm{O}$ & $\begin{array}{l}1615,1624 \\
1631\end{array}$ & document & $\begin{array}{l}\text { IKDA 4; El. 1624; Man. } \\
1631\end{array}$ \\
\hline $\begin{array}{l}\text { Piotr Bohdanowicz } \dagger \\
1636 / 8\end{array}$ & $\mathrm{O}$ & 1620 & pomiannik & $\begin{array}{l}\text { Drevnij pomiannik..., } \\
\text { op. cit., p. } 65\end{array}$ \\
\hline $\begin{array}{l}\text { Ostafi Bohdanowicz } \dagger \\
1624 / 8\end{array}$ & $\mathrm{O}$ & 1620,1624 & $\begin{array}{l}\text { pomiannik, } \\
\text { document }\end{array}$ & op. cit., p. 65; El. 1624 \\
\hline $\begin{array}{l}\text { Paweł Bohdanowicz } \dagger \text { after } \\
1618\end{array}$ & ? & & & \\
\hline $\begin{array}{l}\text { Bohdan Bohdanowicz } \dagger \\
\text { after } 1609\end{array}$ & $?$ & & & \\
\hline $\begin{array}{l}\text { Iwan Fedorowicz } \dagger \text { before } \\
1640\end{array}$ & $\mathrm{O}$ & 1634 & document & AJZR 8-3, p. 530 \\
\hline Abram Ostafiowicz - & $\mathrm{O}$ & & report & $\begin{array}{l}\text { J. Jerlicz, op. cit., vol. } 1 \text {, } \\
\text { p. } 150\end{array}$ \\
\hline
\end{tabular}




\begin{tabular}{|c|c|c|c|c|}
\hline Name & 1 & 2 & 3 & 4 \\
\hline Michał Ostafiowicz - & $\mathrm{O}$ & 1642 & report & $\begin{array}{l}\text { J. Jerlicz, op. cit., vol. } \\
\text { 1, p. } 46\end{array}$ \\
\hline Stefan Daniłowicz - & $\mathrm{O}$ & 1632 & literature & $\begin{array}{l}\text { M.A. Maksimovicz, op. } \\
\text { cit., vol. 1, p. } 302\end{array}$ \\
\hline $\begin{array}{l}\text { Mikołaj Daniłowicz } \dagger \\
\text { before } 1640\end{array}$ & $?$ & & & \\
\hline Stefan Pawłowicz - & $?$ & & & \\
\hline \multicolumn{5}{|l|}{ Strzyżowski } \\
\hline Gniewosz until 1602 & $?$ & & & \\
\hline \multicolumn{5}{|l|}{ Suryn } \\
\hline $\begin{array}{l}\text { Andrzej Niemirowicz } \dagger \\
\text { after } 1581\end{array}$ & $?$ & & & \\
\hline $\begin{array}{l}\text { Hordej Niemirowicz } \dagger \\
\text { before } 1598\end{array}$ & $?$ & & & \\
\hline $\begin{array}{l}\text { Harasym Stanisławowicz } \\
\dagger 1613\end{array}$ & $\mathrm{O}$ & & & \\
\hline $\begin{array}{l}\text { Potej Stanisławowicz } \dagger \\
1595\end{array}$ & $\mathrm{O}$ & & & \\
\hline $\begin{array}{l}\text { Iwan Harasymowicz } \dagger \\
\text { before } 1613\end{array}$ & $\mathrm{O}$ & & & \\
\hline $\begin{array}{l}\text { Wasyl Harasymowicz } \dagger \\
\text { before } 1613\end{array}$ & $\mathrm{O}$ & & & \\
\hline $\begin{array}{l}\text { Andrzej Harasymowicz } \dagger \\
1619\end{array}$ & $\mathrm{O}$ & & & \\
\hline Piotr Iwanowicz - & $\mathrm{O}$ & 1619 & document & List 1619 \\
\hline Adam Wasylowicz - & $\mathrm{O}$ & 1647 & document & El. 1647 Kossow \\
\hline Aleksander Andrzejowicz - & $\mathrm{O}$ & 1647 & document & El. 1647 Kossow \\
\hline Mikołaj Andrzejowicz - & $?$ & & & \\
\hline Rafał Andrzejowicz - & $\mathrm{O}$ & before 1630 & & \\
\hline--- & $\mathrm{C}$ & 1630 & $\begin{array}{l}\text { report, } \\
\text { document }\end{array}$ & Opis, vol. 14, p. 58 \\
\hline Jan Andrzejowicz & $\mathrm{O}$ & 1647 & & El. 1647 Tryzna \\
\hline \multicolumn{5}{|l|}{ Szaszkiewicz } \\
\hline Wasyl † 1594 & $\mathrm{O}$ & & & \\
\hline $\begin{array}{l}\text { Mikołaj Wasylowicz until } \\
1622\end{array}$ & $\mathrm{O}$ & until $\dagger$ & document & $\begin{array}{l}\text { Sbornik matierialov..., } \\
\text { op. cit., p. } 43\end{array}$ \\
\hline
\end{tabular}




\begin{tabular}{|c|c|c|c|c|}
\hline Name & 1 & 2 & 3 & 4 \\
\hline $\begin{array}{l}\text { Fedor Wasylowicz unil † } \\
\text { before } 1618\end{array}$ & $\mathrm{O}$ & until $\dagger$ & report & $\begin{array}{l}\text { J. Jerlicz, op. cit., vol. } \\
\text { 1, p. } 86\end{array}$ \\
\hline \multicolumn{5}{|l|}{ Szyszko-Stawecki } \\
\hline Iwan $\dagger 1585$ & $\mathrm{O}$ & & & \\
\hline Semen $\dagger$ before 1583 & $?$ & & & \\
\hline $\begin{array}{l}\text { Roman Semenowicz } \dagger \\
1585\end{array}$ & $?$ & & & \\
\hline Semen Iwanowicz $† 1595$ & ? & & & \\
\hline Michał Iwanowicz $† 1592$ & $\mathrm{O}$ & & & \\
\hline Fedor Iwanowicz $\dagger 1629$ & $\mathrm{O}$ & & & \\
\hline $\begin{array}{l}\text { Hryhory Romanowicz } \dagger \\
1617\end{array}$ & ? & & & \\
\hline Hryhory Semenowicz - & C & 1649 & report & $\begin{array}{l}\text { WAPK, Archiwum Dóbr } \\
\text { Pomorzany, no. 89, 1. } 25\end{array}$ \\
\hline $\begin{array}{l}\text { Paweł Michałowicz } \dagger \text { after } \\
1633\end{array}$ & $\mathrm{O}$ & 1633 & report & $\begin{array}{l}\text { Golubev - Mogiła, p. } \\
\text { 528; BUAN, Ms. } 21979\end{array}$ \\
\hline Iwan Michałowicz $† 1610$ & $\mathrm{O}$ & & & \\
\hline Semen Fedorowicz - & $\mathrm{O}$ & 1615,1647 & document & $\begin{array}{l}\text { IKDA, 9; El. } 1647 \\
\text { Tryzna }\end{array}$ \\
\hline Hryhory Fedorowicz - & $\mathrm{O}$ & 1632 & literature & $\begin{array}{l}\text { W. Kaczorowski, Sejmy } \\
\text { konwokacyjny i elekcyjny } \\
\text { w okresie bezkrólewia } \\
1632 \text { r., Opole, } 1986, \\
\text { p. } 176\end{array}$ \\
\hline Paweł Fedorowicz - & $?$ & & & \\
\hline \multicolumn{5}{|l|}{ Trypolski } \\
\hline $\begin{array}{l}\text { Fedor Wasylowicz } \dagger \text { after } \\
1609\end{array}$ & $\mathrm{O}$ & & & \\
\hline $\begin{array}{l}\text { Hapon Wasylowicz } \dagger \text { after } \\
1613\end{array}$ & $\mathrm{O}$ & & & \\
\hline \multicolumn{5}{|l|}{$\begin{array}{l}\text { Żdan Wasylowicz } \dagger \text { after } \\
1609\end{array}$} \\
\hline $\begin{array}{l}\text { Teodor (Fedor junior) } \\
\text { Wasylowicz } \dagger \text { after } 1618\end{array}$ & $\mathrm{O}$ & & & \\
\hline \multicolumn{5}{|l|}{$\begin{array}{l}\text { Andrzej Fedorowicz } \dagger \\
1618 / 24\end{array}$} \\
\hline $\begin{array}{l}\text { Matwiej Fedorowicz } \dagger \\
1634 / 40\end{array}$ & $\mathrm{O}$ & & & \\
\hline
\end{tabular}


DENOMINATIONAL COMPOSITION OF THE KIEVAN...

\begin{tabular}{|c|c|c|c|c|}
\hline Name & 1 & 2 & 3 & 4 \\
\hline $\begin{array}{l}\text { Herman Fedorowicz } \dagger \\
1634 / 40\end{array}$ & $\mathrm{O}$ & 1624 & & El. 1624 \\
\hline $\begin{array}{l}\text { Maksym Fedorowicz } \dagger \\
1634 / 40\end{array}$ & $\mathrm{O}$ & 1615,1624 & & IKDA 9; El. 1624 \\
\hline Marek Fedorowicz $† 1649$ & $\mathrm{O}$ & 1624,1631 & & El. 1624; Man. 1631 \\
\hline $\begin{array}{l}\text { Parfen Haponowicz } \dagger \text { after } \\
1647\end{array}$ & $\mathrm{O}$ & 1631,1647 & & $\begin{array}{l}\text { Man. 1631; El. } 1647 \\
\text { Tryzna }\end{array}$ \\
\hline $\begin{array}{l}\text { Fedor Haponowicz } \dagger \text { after } \\
1640\end{array}$ & $\mathrm{O}$ & 1624,1631 & & El. 1624; Man. 1631 \\
\hline $\begin{array}{l}\text { Nikifor Haponowicz } \dagger \\
\text { after } 1629\end{array}$ & $\mathrm{O}$ & & & \\
\hline $\begin{array}{l}\text { Iwan (Jan) Teodorowicz } \dagger \\
\text { after } 1640\end{array}$ & $\mathrm{O}$ & 1624,1629 & & El. 1624; Prot. 1629 \\
\hline Fedor Teodorowicz - & $\mathrm{O}$ & 1647 & & El. 1647 Kossow \\
\hline \multicolumn{5}{|l|}{ Ostafi Teodorowicz - } \\
\hline \multicolumn{5}{|l|}{ Eliasz Andrzejowicz - } \\
\hline Wasyl Matwiejowicz - & $\mathrm{O}$ & 1629 & & Prot. 1629 \\
\hline \multicolumn{5}{|l|}{ Iwan Matwiejowicz - } \\
\hline Hrehory Matwiejowicz - & $\mathrm{O}$ & 1632 & & $\begin{array}{l}\text { M.A. Maksimowič, vol. } \\
\text { 2, p. } 202\end{array}$ \\
\hline \multicolumn{5}{|l|}{ Matwiej Hermanowicz - } \\
\hline \multicolumn{5}{|l|}{ Piotr Hermanowicz - } \\
\hline Aleksander Hermanowicz - & $\mathrm{O}$ & 1647 & & El. 1647 Tryzna \\
\hline \multicolumn{5}{|l|}{ Wacław Fedorowicz - } \\
\hline Iwan (Jan) Fedorowicz - & $\mathrm{O}$ & 1647 & & El. 1647 Tryzna \\
\hline \multicolumn{5}{|l|}{ Kostiuk Fedorowicz - } \\
\hline \multicolumn{5}{|l|}{ Aleksander Parfenowicz - } \\
\hline \multicolumn{5}{|l|}{ Józef Parfenowicz - } \\
\hline Hapon Nikiforowicz? - & $\mathrm{O}$ & 1647 & & BUAN, Ms. 23368 \\
\hline Stefan Nikiforowicz? - & $\mathrm{O}$ & 1647 & & El. 1647 \\
\hline \multicolumn{5}{|l|}{ Tysza-Bykowski } \\
\hline Fedor $\uparrow 1572$ & $\mathrm{O}$ & & & \\
\hline $\begin{array}{l}\text { Aleksander Fedorowicz } \dagger \\
1613\end{array}$ & $\mathrm{O}$ & & & \\
\hline Wasyl Fedorowicz $† 1624$ & $?$ & & & \\
\hline
\end{tabular}




\begin{tabular}{|c|c|c|c|c|}
\hline Name & 1 & 2 & 3 & 4 \\
\hline $\begin{array}{l}\text { Adam Aleksandrowicz } \dagger \\
1636\end{array}$ & $\mathrm{O}$ & 1636 & report & $\begin{array}{l}\text { J. Jerlicz, op. cit., vol. } \\
\text { 1, p. } 43\end{array}$ \\
\hline $\begin{array}{l}\text { Jeremiasz Aleksandrowicz } \\
\dagger 1647\end{array}$ & $\mathrm{O}$ & 1647 & report & ibid., p. 60 \\
\hline Fedor Wasylowicz $† 1644$ & $\mathrm{C}$ & 1644 & report & ibid., p. 47 \\
\hline Wasyl Wasylowicz $† 1634$ & $?$ & & & \\
\hline Gabriel Adamowicz - & $?$ & & & \\
\hline Paweł Adamowicz - & $?$ & & & \\
\hline Jan Adamowicz - & $\mathrm{O}$ & 1632 & literature & $\begin{array}{l}\text { M.A. Maksimovič, vol. } \\
\text { 2, p. } 203\end{array}$ \\
\hline Adam Jeremiowicz - & $\mathrm{O}$ & 1632 & literature & ibid. \\
\hline Jan Fedorowicz $† 1646$ & C & & & \\
\hline Michał Fedorowicz - & $\mathrm{C}$ & & & \\
\hline Gabriel Fedorowicz - & $\mathrm{C}$ & & & \\
\hline \multicolumn{5}{|l|}{ Tyszkiewicz-Łohojski } \\
\hline Jur (Jerzy) † 1576 & $\mathrm{O}$ & 1576 & literature & $\begin{array}{l}\text { Viktorovskij, 1911, no. } \\
\text { 2; H. Lulewicz, Skład..., } \\
\text { op. cit., p. } 428\end{array}$ \\
\hline $\begin{array}{l}\text { Frydrych (Fryderyk) } \\
\text { Jurowicz } † 1621\end{array}$ & $\mathrm{O}$ & 1601 & document & Man. 1601 \\
\hline--- & $\mathrm{U}$ & 1603 & document & $\begin{array}{l}\text { Svodnaia, p. } 16 ; \\
\text { Viktorovskij, } 1911 \text {, no. } 2\end{array}$ \\
\hline $\begin{array}{l}\text { Ostafi (Jan Eustachy) } \\
\text { Jurowicz } † 1631\end{array}$ & $\mathrm{O}$ & & literature & op. cit. \\
\hline--- & C & 1622 & document & ARSI Pol. 77, 1. 69-70 \\
\hline Marcin Jurowicz $† 1595$ & $\mathrm{O}$ & 1595 & literature & Viktorovskij, 1911, no. 2 \\
\hline Janusz Frydrychowicz - & $\mathrm{C}$ & 1628,1630 & $\begin{array}{l}\text { literature, } \\
\text { report }\end{array}$ & $\begin{array}{l}\text { Klasztory } \\
\text { Bernardyńskie..., op. } \\
\text { cit., p. } 215 \text {; Opis, vol. } \\
\text { 14, p. } 61\end{array}$ \\
\hline Józef Marcinowicz & $\mathrm{O}$ & & literature & Viktorovskij, 1911 , no. 2 \\
\hline $\begin{array}{l}\text { Stanisław Józefowicz } \dagger \\
1643\end{array}$ & $\mathrm{O}$ & & literature & ibid. \\
\hline--- & $\mathrm{C}$ & 1643 & $\begin{array}{l}\text { literature, } \\
\text { report }\end{array}$ & Opis, vol. 16, p. 7 \\
\hline Antoni Jan Ostafiowicz - & $\mathrm{C}$ & & literature & $\begin{array}{l}\text { Lulewicz, Skład..., op. } \\
\text { cit., p. 437; Viktorovskij, } \\
1911 \text {, no. } 2\end{array}$ \\
\hline
\end{tabular}




\begin{tabular}{|c|c|c|c|c|}
\hline Name & 1 & 2 & 3 & 4 \\
\hline Krzysztof Ostafiowicz - & $\mathrm{C}$ & & literature & $\begin{array}{l}\text { Viktorovskij, 1911, } \\
\text { no. 2; J.M. Giżycki } \\
\text { (Wołyniak), Zniesione } \\
\text { kościoły..., op. cit., p. } \\
168\end{array}$ \\
\hline Felicjan Ostafiowicz - & $\mathrm{C}$ & & literature & Viktorovskij, 1911 , no. 2 \\
\hline \multicolumn{5}{|l|}{ Tyszkiewicz-Skumin } \\
\hline Dymitr until 1576 & $\mathrm{O}$ & until $\dagger$ & literature & $\begin{array}{l}\text { E. Tyszkiewicz, } \\
\text { Groby...,op. cit., p. } 12\end{array}$ \\
\hline Teodor $\dagger 1618$ & $\mathrm{O}$ & 1586,1596 & literature & $\begin{array}{l}\text { Lulewicz, Skład..., op. } \\
\text { cit., p. } 430,432\end{array}$ \\
\hline--- & $\mathrm{U}$ & 1603 & document & Svodnaia, p. 16 \\
\hline Janusz until after 1628 & $\mathrm{U}$ & 1603,1621 & $\begin{array}{l}\text { document, } \\
\text { report }\end{array}$ & ibid., p. 16, 436 \\
\hline \multicolumn{5}{|l|}{ Wielhorski } \\
\hline Wacław $1590-\uparrow 1619$ & ? & & & \\
\hline Piotr $1629-$ & $?$ & & & \\
\hline \multicolumn{5}{|l|}{ Woronicz } \\
\hline Aleksander $\dagger 1611$ & $\mathrm{O}$ & & & \\
\hline $\begin{array}{l}\text { Wasyl Aleksandrowicz } \dagger \\
1613\end{array}$ & $\mathrm{O}$ & & & \\
\hline $\begin{array}{l}\text { Filon Aleksandrowicz } \dagger \\
1639\end{array}$ & $\mathrm{O}$ & $\begin{array}{l}1624,1631 \\
1632\end{array}$ & document & $\begin{array}{l}\text { El. 1624; Man. 1631; } \\
\text { El. } 1632\end{array}$ \\
\hline $\begin{array}{l}\text { Michał (Mikołaj) } \\
\text { Aleksandrowicz } † 1643\end{array}$ & $\mathrm{O}$ & 1643 & $\begin{array}{l}\text { document, } \\
\text { report }\end{array}$ & $\begin{array}{l}\text { BC, Ms. } 2468,1.27 \text {; } \\
\text { Opis, vol. 16, p. } 19\end{array}$ \\
\hline $\begin{array}{l}\text { Fedor Aleksandrowicz } \dagger \\
1646\end{array}$ & $\mathrm{O}$ & $\begin{array}{l}1632,1643, \\
1646\end{array}$ & $\begin{array}{l}\text { document, } \\
\text { report }\end{array}$ & $\begin{array}{l}\text { El. 1632; BC, Ms. } 2468 \text {, } \\
\text { 1. 27; J. Jerlicz, op. cit., } \\
\text { vol. 1, p. } 49\end{array}$ \\
\hline Daniel Jerzy Wasylowicz - & $\mathrm{O}$ & 1622,1647 & document & $\begin{array}{l}\text { Svodnaia, p. 440; El. } \\
1647 \text { Tryzna }\end{array}$ \\
\hline $\begin{array}{l}\text { Dymitr Karol Michałowicz } \\
\text { - }\end{array}$ & ? & & & \\
\hline Samuel Fedorowicz - & $\mathrm{O}$ & 1642,1647 & document & $\begin{array}{l}\text { Golubev - Mogiła, vol. } \\
\text { 2, p. 248; El. } 1647 \\
\text { Kossow }\end{array}$ \\
\hline Michał Fedorowicz - & $\mathrm{O}$ & 1647 & document & El. 1647 Kossow \\
\hline Władysław Fedorowicz - & ? & & & \\
\hline Aleksander Fedorowicz - & ? & & & \\
\hline
\end{tabular}


The above list includes 260 individuals. It consists, however, of 277 positions, because persons who changed their confession in the period under discussion are recorded more than once. I succeeded in ascertaining the confession of 186 persons (72\% of the whole group), who are recorded in 207 entries. In the whole period 1569-1648, 149 nobles of the Orthodox denomination are recorded, 24 dissenters (including 11 Arians) and 30 Catholics (including 4 Uniates). The collected evidence should, however, be analysed from a dynamic perspective, which can be done in several ways.

First, the data will be analysed for several significant points in time. Table 3 compares the number of followers of particular denominations among the Kievan noble families in $1569,1595,1620$ and $1648 .^{20}$

Table 3. The denomination of members of the Kievan noble families active in 1569, 1595, 1620, and 1648

\begin{tabular}{|l|l|l|l|l|l|l|l|l|}
\hline & 1569 & {$[\%]$} & 1595 & {$[\%]$} & 1620 & {$[\%]$} & 1648 & {$[\%]$} \\
\hline Orthodox & 36 & 97,3 & 54 & 93,1 & 59 & 78,7 & 38 & 63,3 \\
\hline Dissenters & 1 & 2,7 & 3 & 5,2 & 10 & 13,3 & 7 & 11,7 \\
\hline Catholic & 0 & 0 & 1 & 1.7 & 6 & 8 & 15 & 25 \\
\hline Total & 37 & 100 & 58 & 100 & 75 & 100 & 60 & 100 \\
\hline
\end{tabular}

Table 4 presents a different approach. Here, the values are compared for periods between the dates set in Table $3 .{ }^{21}$

Table 4. The denomination of members of the Kievan noble families active in periods 1569-95, 1596-1620, and 1620-48

\begin{tabular}{|l|l|l|l|l|l|l|}
\hline & $1569-95$ & $\%$ & $1596-1620$ & $\%$ & $1621-48$ & $\%$ \\
\hline Orthodox & 66 & 91,6 & 83 & 75,45 & 72 & 64,9 \\
\hline Dissenters & 4 & 5.6 & 17 & 15,45 & 14 & 12,6 \\
\hline Catholic & 2 & 2,8 & 10 & 9,1 & 25 & 22,5 \\
\hline Total & 72 & 100 & 110 & 100 & 111 & 100 \\
\hline
\end{tabular}

As indicated by the above comparisons, Catholics were rarely found among the members of Kievan nobles until the Union of Brest, the only attested cases being Aleksander and Mikołaj Siemaszko. Though owners of estates in Kievan voivodeship, they were, however, actually attached to Volyn. Also dissenters found among Kievan nobles were settled either in

20 Included are only the persons whose denomination could have been ascertained. 21 Included are only the persons whose denomination could have been ascertained. 
Lithuania, like Hawryło and Jerofiej Hornostaj as well as Mikołaj Służka, or in Volyn, like Iwan Czaplic-Szpanowski. Families linked closely to Volyn were in the period 1569-95 exclusively Orthodox.

As a result of the religious conflict between the union and 'dis-union', the situation became more dynamic. Between 1596 and 1620, the number of Catholics and dissenters increases, though the followers of the 'greek faith' are still in the majority. Completely Catholicised are in that period Olizar and Tyszkiewicz (both Skumin and Łohojski) families, isolated instances of conversion to Catholicism are found also among Służka, Tysz-Bykowski, and Iwaszeńcewicz-Makarewicz. In the Protestant camp, Hornostaj family remains, as well as some of the Służka, Czaplica, and Iwaszeńcewicz. Arianism starts spreading, accepted by the Babiński family and some members of the Hojski, Niemirycz, and Chreptowicz. The evidence, however, indicates that, in the period 1596-1620, the majority of the noble families remains Orthodox. This concerns following families: Aksak, Butowicz, Jelec, Łaski, Łozek, Pawsza, Proskur, Sołtan, Stecki, Strybel, Suryn, Szaszkiewicz, Szyszko, Trypolski, Woronicz. Also numerous members of Tysza and Niemirycz families remain faithful to the Orthodoxy. Thus, the Kievan noble circles are predominantly Orthodox.

In the last of the sub-periods under discussion, the expansion of the Catholicism intensifies, though the followers of the 'Roman faith' are still in a clear minority. Apart from the already earlier converted Olizar and Tyszkiewicz-Łohojski, no other family turned 'papist' as a whole, although the Aksak and Jelec families are on the verge of such turn. Some families disappear from the political arena of the voivodeship: the Aksaks and Skumin-Tyszkiewicz have sold their estates there, while Siemaszko and Iwaszeńcewicz died out. The Catholic branch of the Tysza-Bykowski family proliferates, and there are isolated sprouts in the family trees of the Łozek (who, however, were soon to die out), Suryn, and Szyszko.

In the initial part of the sub-period under discussion, the Protestant influences remain significant. In the 1640's however, the families that died out: Hojski (1635) and Hornostaj (1637), cannot be easily substituted. Then, the Arianism retains its position thanks to the Babiński, Czaplica, Niemirycz, and Chreptowicz families.

The members of the Orthodox church are in the majority until 1648. The Butowicz, Łaski, Pawsza, Proskur, Sołtan, Stecki, Strybyl, Trypolski, 
and Woronicz families, as well as some of Niemirycz, Suryn, Szyszko, Tysza, and the last of Jelec and Aksak remain true to the faith of their ancestors.

Basing on the collected data related to specific families, one can try to ascertain, with a reasonable probability, the denomination of members of the families under discussion also when there is no direct evidence in this respect. The lack of any evidence of religious diversity within a single family allows us to suggest that brothers followed the same faith (thus we make an assumption concerning all brothers based on data related to one of them). This way, Table 2 may be supplemented with hypothetical data; the result is presented below.

Table 5. The denomination of members of the Kievan noble families active in 1569, 1595, 1620, and 1648 (including hypothetical data)

\begin{tabular}{|l|l|l|l|l|l|l|l|l|}
\hline & 1569 & {$[\%]$} & 1595 & {$[\%]$} & 1620 & {$[\%]$} & 1648 & {$[\%]$} \\
\hline Orthodox & 45 & 95,7 & 64 & 88.9 & 72 & 78,3 & 66 & 73,3 \\
\hline Dissenters & 2 & 4,3 & 5 & 6,9 & 11 & 11,9 & 7 & 7,8 \\
\hline Catholic & 0 & 0 & 3 & 4,2 & 9 & 9,8 & 17 & 18,9 \\
\hline Total & 47 & 100 & 72 & 100 & 92 & 100 & 90 & 100 \\
\hline
\end{tabular}

Table 6. The denomination of members of the Kievan noble families active in periods 1569-95, 1596-1620, and 1620-48 (including hypothetical data)

\begin{tabular}{|l|l|l|l|l|l|l|}
\hline & $1569-95$ & $\%$ & $1596-1620$ & $\%$ & $1621-48$ & $\%$ \\
\hline Orthodox & 82 & 88,2 & 104 & 76,5 & 110 & 71,9 \\
\hline Dissenters & 7 & 7,5 & 19 & 13,9 & 14 & 9,2 \\
\hline Catholic & 4 & 4,3 & 13 & 9,6 & 29 & 18,9 \\
\hline Total & 93 & 100 & 136 & 100 & 153 & 100 \\
\hline
\end{tabular}

These compilations confirm the conclusions proposed above. The lasting predominance of the Orthodox faith in the last of the dates and sub-periods under discussion is even more clearly visible.

The denomination of members of wealthy immigrant families

It would be interesting to supplement the data related to the Ruthenian nobles with information on incoming Polish families that married into wealthy Kievan gentry, acquired Ukrainian estates and started to play an important role in the local political arena. 
Table 7 . The denomination of members of wealthy incoming gentry, Kievan voivodeship 1569-1648 22

\begin{tabular}{|c|c|c|c|c|}
\hline 1 & 2 & 3 & 4 & 5 \\
\hline \multicolumn{5}{|l|}{$\begin{array}{l}\text { Charlęski (Charliński) } \\
\text { Kievan branch, before } 1569\end{array}$} \\
\hline Szczęsny $\dagger 1601$ & ? & & & \\
\hline $\begin{array}{l}\text { Stanisław, son of Szczęsny } \\
\dagger 1628\end{array}$ & $?$ & & & \\
\hline $\begin{array}{l}\text { Mikołaj, son of Szczęsny } \dagger \\
1623\end{array}$ & $\mathrm{C}$ & 1622,1623 & $\begin{array}{l}\text { document, } \\
\text { literature }\end{array}$ & $\begin{array}{l}\text { AGAD, Dokumenty } \\
\text { Pergaminowe 6116; SGKP, } \\
\text { 'Byszów' }\end{array}$ \\
\hline Jerzy, son of Szczęsny $† 1617$ & $\mathrm{C}$ & 1600 & literature & $\begin{array}{l}\text { J.M. Giżycki, Wykaz } \\
\text { klasztorów..., p. } 21\end{array}$ \\
\hline $\begin{array}{l}\text { Szczęsny, son of Stanisław } \\
\dagger 1648\end{array}$ & ? & & & \\
\hline Samuel, son of Jerzy $† 1644$ & $\mathrm{C}$ & 1644 & literature & SGKP, 'Byszów’ \\
\hline \multicolumn{5}{|l|}{$\begin{array}{l}\text { Witowski of Jastrzębiec, } \\
1578\end{array}$} \\
\hline Rafał $\dagger 1622 / 23$ & $?$ & & & \\
\hline Łukasz $† 1647$ & $\mathrm{C}$ & 1628 & literature & $\begin{array}{l}\text { J.M. Giżycki, Wykaz } \\
\text { klasztorów..., p. } 21\end{array}$ \\
\hline \multicolumn{5}{|l|}{$\begin{array}{l}\text { Frąckiewicz-Radzimiński, } \\
1581\end{array}$} \\
\hline Michał $\dagger$ after 1607 & $?$ & & & \\
\hline Mikołaj † 1630 & $\mathrm{P}$ & 1621 & literature & $\begin{array}{l}\text { H. Wisner, Rzeczpospolita } \\
\text { Wazów. Czasy Zygmunta III } \\
i \text { Wtadystawa IV, Warsaw, } \\
\text { 2002, p. } 84\end{array}$ \\
\hline $\operatorname{Jan} \dagger 1631$ & $?$ & & & \\
\hline \multicolumn{5}{|l|}{ Stużyński, 1581} \\
\hline Kasper $\dagger$ after 1592 & $\mathrm{C}$ & 1570 & document & BUAN, Ms. 23283, 1.3 \\
\hline Mierzwiński, 1583 & & & & \\
\hline
\end{tabular}

22 The table contains data pertaining to members of those families of Polish and Lithuanian origin that attained a high material and political status in the Kiev voivodeship. The classification is based on data collected in Litwin, Naptyw szlachty polskiej na Ukraine 15569-1648, Warsaw, 2000, passim (landowners belonging to the second and third economic group as well as landed officials). Omitted are magnates, who had any relations with the borderlands only through their representatives or during military campaigns: Jan and Stanisław Daniłowicz, Stanisław and Aleksander Koniecpolski, Stanisław, Jerzy and Konstanty Lubomirski, Jakub, Jan, and Marek Sobieski, Tomasz and Jan Zamoyski, Stanisław Źółkiewski. The families are listed here in a sequence corresponding to their respective arrivals to Kievan voivodeship. The dates given at the family names indicate the first attestations in the Kievan region. The sequence of columns repeats that of Table 2 . 


\begin{tabular}{|c|c|c|c|c|}
\hline 1 & 2 & 3 & 4 & 5 \\
\hline Prokop $†$ after 1605 & ? & & & \\
\hline $\begin{array}{l}\text { Andrzej, son of Prokop } \dagger \\
1624 / 8\end{array}$ & ? & & & \\
\hline $\begin{array}{l}\text { Andrzej, son of Andrzej } \dagger \\
\text { after } 1648\end{array}$ & $?$ & & & \\
\hline \multicolumn{5}{|l|}{ Ogonowski, 1592} \\
\hline Jan $†$ after 1607 & $?$ & & & \\
\hline \multicolumn{5}{|l|}{ Leszczyński, 1600} \\
\hline Andrzej $† 1606$ & $\mathrm{P}$ & & literature & $P S B$, vol. 17, pp. 101-03 \\
\hline Rafał $\dagger 1644$ & $\mathrm{P}$ & & literature & $P S B$, vol. 17, pp. $135-36$ \\
\hline \multicolumn{5}{|l|}{ Złotopolski, 1602} \\
\hline Piotr $\dagger 1629$ & $?$ & & & \\
\hline \multicolumn{5}{|l|}{ Błędowski, 1606} \\
\hline Jan $† 1621 / 5$ & $\mathrm{P}$ & 1613 & literature & $\begin{array}{l}\text { J. Byliński, Dwa sejmy } \\
\text { z roku 1613, Wrocław, } \\
1984 \text {, p. } 250\end{array}$ \\
\hline \multicolumn{5}{|l|}{ Drzewiecki, 1612} \\
\hline Jan $† 1639 / 40$ & $\mathrm{P}$ & 1624 & document & El. $1624 ; A J Z R, 1 / 7$, p. 88 \\
\hline \multicolumn{5}{|l|}{ Abramowicz, 1624} \\
\hline Mikołaj † 1651 & $\mathrm{P}$ & 1651 & literature & $P S B$, vol. 1 , pp. $14-15$ \\
\hline \multicolumn{5}{|l|}{ Wielam (Wilam), 1624} \\
\hline Stanisław $† 1638$ & $\mathrm{P}$ & $\begin{array}{l}1625,1631, \\
1635\end{array}$ & document & $\begin{array}{l}\text { Akta synodów..., } \\
\text { op. cit., 471, } 557 \text {, } \\
\text { J.H. Drohojowski, op. cit., } \\
\text { p. } 112\end{array}$ \\
\hline \multicolumn{5}{|l|}{ Firlej, 1628} \\
\hline Andrzej $† 1649$ & $\mathrm{P}$ & 1649 & literature & $P S B$, vol. 6, p. 476 \\
\hline \multicolumn{5}{|l|}{ Łaszcz-Tuczapski, 1628} \\
\hline Samuel & $\mathrm{C}$ & 1628 & literature & $\begin{array}{l}\text { J.M. Giżycki, Wykaz } \\
\text { klasztorów..., p. } 203\end{array}$ \\
\hline \multicolumn{5}{|l|}{ Zborowski, 1628} \\
\hline Andrzej † 1630 & $\mathrm{C}$ & & literature & $\begin{array}{l}\text { W. Sokołowski, Politycy } \\
\text { schytku Ztotego Wieku. } \\
\text { Matopolscy przywódcy } \\
\text { szlachty i parlamentarzyści } \\
\text { w latach 1574-1605, } \\
\text { Warsaw, 1997, p. } 102\end{array}$ \\
\hline $\begin{array}{l}\text { Marcjan, son of Andrzej } \dagger \\
1639\end{array}$ & $\mathrm{C}$ & & & \\
\hline $\begin{array}{l}\text { Hieronim, son of Piotr, } \dagger \\
\text { after } 1649\end{array}$ & $?$ & & & \\
\hline Wojnarowski, 1630 & & & & \\
\hline
\end{tabular}




\begin{tabular}{|c|c|c|c|c|}
\hline 1 & 2 & 3 & 4 & 5 \\
\hline Stefan $\dagger 1646 / 47$ & A & 1645 & document & $A J Z R 2 / 1$, p. 281 \\
\hline \multicolumn{5}{|c|}{ Potocki of Lubicz, 1636} \\
\hline Florian $\dagger 1641 / 42$ & $\mathrm{C}$ & & literature & $\begin{array}{l}\text { B.J. Wanat, Zakon } \\
\text { Karmelitów..., op. cit., p. } \\
379\end{array}$ \\
\hline \multicolumn{5}{|l|}{ Drohojowski, 1636} \\
\hline Andrzej $† 1642$ & $\mathrm{P}$ & 1632,1635 & report & $\begin{array}{l}\text { Akta synodów..., op. cit., } \\
580 ; \\
\text { J.H. Drohojowski, op. cit., } \\
\text { p. } 112\end{array}$ \\
\hline \multicolumn{5}{|c|}{$\begin{array}{l}\text { Charlęski (Charliński), Volyn } \\
\text { branch, } 1638\end{array}$} \\
\hline Hieronim $\dagger 1641$ & $\mathrm{C}$ & 1632 & document & $\begin{array}{l}\text { Volumina Legum, vol. } 3 \text {, } \\
\text { p. } 366\end{array}$ \\
\hline Krzysztof, after 1648 & ? & & report & $\begin{array}{l}\text { A.S. Radziwiłł. Pamiętnik } \\
\text { o dziejach w Polsce, ed. by } \\
\text { A. Przyboś, R. Żelewski, } \\
\text { vols. 1-3, Warsaw, } 1980 \text {, } \\
\text { vol. 1, pp. 201, } 210\end{array}$ \\
\hline \multicolumn{5}{|c|}{$\begin{array}{l}\text { Kazimierski from Biberstein, } \\
1640\end{array}$} \\
\hline Tomasz $\dagger 1667$ & A & 1667 & literature & PSB, vol. 12, p. 295 \\
\hline \multicolumn{5}{|l|}{ Dzik 1643} \\
\hline $\operatorname{Jan} \dagger 1651$ & $\mathrm{O}$ & & literature & $\begin{array}{l}\text { Ukraina..., op. cit.. Dz. III, } \\
\text { p. } 546\end{array}$ \\
\hline \multicolumn{5}{|l|}{ Pogórski, 1644} \\
\hline Jan $†$ after 1648 & $?$ & & & \\
\hline \multicolumn{5}{|c|}{$\begin{array}{l}\text { Krzętowski (Krętowski), } \\
1648\end{array}$} \\
\hline Jan $† 1653 / 5$ & $\mathrm{C}$ & & & \\
\hline \multicolumn{5}{|c|}{$\begin{array}{l}\text { Zamoyski (non-entailer line), } \\
1639\end{array}$} \\
\hline Aleksander $\dagger 1655$ & $?$ & & & \\
\hline
\end{tabular}

Among 38 persons listed above, the denomination of 23 can be identified. There are 11 Catholics, 8 Calvinists, 2 Arians, and 2 Orthodox. For 15 persons such identification was impossible. Catholics were most probably in the majority among them (almost certainly Aleksander Zamoyski, Krzysztof Charlęski, and Rafał Witowski). However, the numerous presence of immigrant adherents of other denominations (to those already established probably Michał and Jan Frąckiewicz-Radziniński should be 
added) precludes identification of all incomers as Catholics. ${ }^{23}$ Evidently, the inflow of wealthy Polish nobles to Kiev voivodeship did not necessarily enhance the Catholic presence in those lands.

The denomination of members of Kievan gentry In order to compile a more complete picture of the religious structure of the Kievan nobility, we need at least approximately ascertain the religious composition of the Ruthenian gentry, constituting a majority among the local szlachta. Any more detailed analysis is here as difficult as it is in the case of noble families. Kievan gentry formed an even larger group, and the histories of individual families were only rarely studied in depth. To give a more complete picture of the religious composition of that group, I include herewith data pertaining to the denomination of members of the Ruthenian gentry families who owned estates in the Kievan region in 1569-1648. Included are both local (that is, settled before 1569) and immigrant (after 1569) families; the whole list comprises 179 entries. ${ }^{24}$ For 62 families there is no information indicating their faith. ${ }^{25}$ On the other hand, there are sources evidencing that members of 111 families

23 Doubts may arise, for example, when immigrant gentry marries into Protestant or Orthodox families. This is the case of Feliks Charlęski, married first with Fenna Lubecka (Orthodox), and then with Katarszyna Zbaraska (Arian). Additionally, the lack of any Catholic foundations during his activity may be significant, despite of his impressive wealth and the scarcity of 'roman' churches in the Kiev region. It seems probable that he died as a Catholic (SGKP, Byszów). Recently, N. Jakowenko ('Relihijni konversiji...', op. cit., p. 76) questioned the Catholic faith of Feliks Charlęski. Her main argument - the marriage between Feliks and a Protestant, Helena Dorohostajska (who was later accused of poisoning her husband) - may be challenged. The person in question is Feliks Charlęski, son of Mikołaj, and thus a nephew of the Kievan chamberlain. Dorohostajska could not be identified as a wife of the latter, because it is Katarzyna de domo Zbaraska, who was repeatedly mentioned as Kievan chamberlain's widow; see, for example, Ukraina, op. cit., Dz. II, pp. 147, 243, 493, 609.

24 The list is based on data collected by N. Jakowenko, Ukrajins'ka šljachta, op. cit., pp. 147-50, and the material collected by the present author, see Litwin, Naptyw, op. cit. 25 Bahrynowski, Bałakier, Basarab, Bech, Biłyj, Bliszczanowicz-Werowka, Bobojeda, Bołsunowski, Boruchowski, Budyło, Czechowicz-Orelski, Czyrski, Daboszyński, Drabowicz-Osdzitowski, Hroza-Chowański, Hrynkowicz, Hubiński, Iskorostyński, Jełcza-Rusinowski, Kiełbowski, Koczurowski, Korkodynowicz-Sypnowski, Korkoszka, Kożuchowski, Krasnodworski, Kreczetowski, Kryzyn-Żukowiecki, Ladowicz, Ludwiski, Łuczkiewicz-Kochanowski, Małyszka, Mazepa-Koledyński, Merezko, Moczulski, Mokreński, Mormył, Niedaszkowski, Ometyński, Ostałecki, Ostanko, Ostrowski, Paszkiewicz, Perocki, Pieslak, Pokalewski, Postołowski, Przybuski, Rozsudowski, 
remained true to the Orthodox faith in the period under discussion. Protestant denominations were less popular among Ruthenian gentry than they were among nobles. Calvinists are found in two families only, and Arians - in four families. Neither was Catholicism well received among that group; it's in seven families only that Roman Catholics are found, and Uniates - in four families.

List 1. Orthodox members of Ruthenian gentry families in the Kiev voivodeship, 1569-164826

Andruski, Zosima monk 1674, Bajbuza, Hrehory 1617, Fedor 1624, Symeon 1630, Baranowicz (Baranowski) (b.), Stefan 1699, Bereżecki, Teodosij monk 1612, Wasyl 1632, Zachariasz 1632, Fedor clergyman 1631, 1648, Biełkiwicz (Bielikiewicz), Iwan 1614, Biłoszycki, Dymitr 1682, Maryna 1680, Bobicki, Jan 1646, Brzozowski, Iwan 1618, Maksymilian 1632, 1647, Buthak (Buthakowski), Komon 1694, Bykowski, Mikołaj 1632, Janusz 1632, Chalepski, Konstanty 1699, Abram 1699, Chałaim, numerous 1624, Andrzej 1640, Chłusowicz, Roman 1624, Chodakowski, Teodor 1679, Michał 1688, Hrehory 1688, Jan 1701, Iwan $† 1688$, Chodyka-Krenicki, Teodor 1615, Czerchawski, Hrehory clergyman $\dagger$ 1613, Jan 1631, Andrzej 1632, Czerkas, Jan 1624, Czernik, Hrehory 1619, 1632, Czopowski, Samuel 1688, Czudowski, Wasyl 1633, Dachnowicz, Mikołaj 1624, Jarosz 1624, Dąbrowski (Dembrowski), Wasyl 1631, Piotr 1641, Diwoczka, Onisyfor clergyman † 1588, Dolmatowicz-Isajkowski, Iwan 1619, Dubicki (Dubnicki), Rafał 1624, Mikołaj 1629, Jan 1647, Dublański, Samuel 1647, Marianna 1681, Dumiński, Daniel 1633, 1640, 1647, Mikołaj $\dagger 1689$, Dzietkowski, Warłaam clergyman 1640, 1647, numerous 1682, 1688, 1689, Fedorowicz, Filip 1624, Łukasz 1629, Golimunt, Makary clergyman 1617, Hajewski-łowdykowski, Fedor 1680, Maksymilian † 1710, Hański, Aleksander 1633, Hołowiński, Tymofiej 1634, Anna $=$ Kropiwnicki 1640, $\uparrow 1648$, Dymitr, Samuel, Konstanty 1679, Hołub, Jerzy 1631, Daniel 1647, Horain, Jan 1612, 1624, Hrazny, Borys 1647, Hryczyna, Jan 1642, Hulkiewicz-Hlebowski, Filon 1625, Iwan 1632, Humieniecki, Jerzy 1624, Agafia 1632, 1636, Iliński, Filip 1584, Fedor 1691, Jarmoliński, Mikołaj 1601, Jan 1625, 1637, 1646, Serafina

Sebastianowicz, Synhur, Szawuła, Traska, Uchłowski, Urucki, Werbowiecki, Wielimont, Wityński (Wituński), Zakusiło, Zbranny, Zubryk, Żwan-Deleszkan, Żubr.

26 I have included here also data pertaining to later periods, assuming ancestors of Orthodox persons were of the same faith; see above, note 17 . 
monk 1646, Jenicz-Komicki, Trochim 1648, Jan 1649, Jerlicz, Maciej, Joachim 1647, Jan, Jarosz 1679, Kaleński, Michał 1685, Jan 1699, Karwowski, Aleksander 1667, 1671, Kierdanowski, Fedor 1629, Piotr 1679, Kiewlicz, Krzysztof 1596, Hrehory 1624, Krzysztof 1653, Kniehyniński, Tymofiej 1632, Mikołaj 1679, Kopeć (Koptewicz), n. 1645, Owdotia = 1 Kropiwnicka, = 2 Kędzierska, Korczowski, Iwan 1598, Kostiuszkowski, Eliasz, Jan 1699, Kotłubaj-Iłiaszewski, Wasyl, Teodor 1585, Kozarewski, Stefan until 1700, Kubiliński, Eliasz 1682, Lasota, Michał 1624, Jerzy 1629, Ostafi 1631, Lemiesz, Benedykt 1647, Lew, Stefan 1647, Lewkowski, Suprun 1631, Michał 1630, numerous 1688, 1699, 1715, Liplański, Daniel 1612, Hrehory 1647, Macharyński, Helena = Kaznowska 1648, Michał 1654, Maluszycki, Andrzej 1624, Mieleniewski, numerous 1682, 1688, 1699, Mirowicki, n. 1636, Moszczenicki, Jerzy 1631, Moszkowski, Tyszko 1660, Maciej 1688, Myszka-Chołoniewski, Michał 1619, Semen 1637, n = Kropiwnicki † 1648, Neczaj-Hruzewicz, Iwan 1624, Nestorycki, Iwan 1631, Niekraszewicz, Samuel 1631, Niemirowicz-Szkarewski, Teodor 1622, Mikołaj 1625, Niepituszczy, Roman 1577, Rafał clergyman 1613, 1618, Niewmirycki, Fedor, Iwan 1629, Roman 1647, Pelagia 1647, Stefan 1680, Aleksander, Jan 1699, Ohij-Tyszkiewicz, Jan 1617, Aleksander 1632, Oleksicz-Gieranowski, Aleksander 1631, 1632, 1647, Bohufał 1647, Paszyński, Terech 1629, Daniel 1631, Pawłowicz, Demian, Prokop, Szymon 1601, Konstanty, Michał 1624, Jaków 1666, Peresecki, Martyn clergyman 1646, 1647, Pilipowski (Pilipowicz), Paweł 1647, Poźniak, Anna = Arciszewski 1640, Rabsztyński, Aleksander 1645, Raj, Filon 1572, Redczyc, Tomasz 1719, Rodkiewicz, Hipolit 1624, Sierchowiecki, Jan 1624, Silicz, Mikołaj 1628, Nikodem clergyman 1636, 1640, Krzysztof 1654, Siła-Nowicki, Teodor 1655, Skipor, Adam 1647, Skurat, Marek 1681, Stefan 1687, Piotr 1691, Maciej 1699, Sokołowski, Iwan 1629, Aleksander 1647, 1649, Sokor, Eustachy, 1624, Roman 1624, Samuel 1631, Hrehory 1645, Sołoma, Jan 1612, Staszkiewicz, Jan 1631, Strzelnicki, Ignacy 1647, Jan 1679, Susło (Susło-Żerbiłło), Mikołaj 1631, Teodor 1632, 1637, Jan 1667, Jakub, Andrzej 1679, Synhajewski, Samuel, Paweł 1699, Michał 1720, Szczeniewski, Marcin 1624, Daniel, Paweł 1647, Krzysztof 1679, Szostakowski, numerous 1686, Świacki, Semen 1614, Tretiak, Stanisław 1624, 1631, 1640, 1647, Aleksander 1698, Uszczap (Uszczapowski), Michał, Hrehory, Artem, Jewchim 1690, Waśkowski, Stefan, Jewtych 1682, Wereszczaka, Fedor 1624, Prokop 1647, Wigura, Stanisław 1624, Semen 1647, 1649, Wołkowicz (Wołkowski), 
Andrzej 1647, Paweł, Antoni, Jan 1684, Wyhowski, Iwan 1619, Bohdan 1629, Ostafi, Fedor 1631, Iwan 1638, Piotr 1647, Jakub, Łukasz, Teodor 1679, Bazyli, Samuel 1698, Wyszpolski, Benedykt, Mikołaj Stefan 1699, Zdzitowiecki, Jan 1618, Zołotoliński, Eliasz 1647, Zub, Bazyli 1664, Żaszkowski, n. 1649, Żmijowski, Jan 1596, Wacław 1624.

List 2. Dissenter members of Ruthenian gentry families in the Kiev voivodeship, 1569-1648

Calvinists: Dachnowicz, Mikołaj 1642, Motowidło-Bieniewicz, Wacław 1635.

Arians: Czudowski, numerous before 1648, Jenicz-Bystrycki, Iwan, Ilia, Paweł 1632-48, Peresecki, Piotr, Andrzej, Anna 1611, Rostkowski, Mikołaj, Stefan 1632.

List 3. Catholic members of Ruthenian gentry families in the Kiev voivodeship, 1569-1648

Roman Catholics: Chodyka-Krenicki, Teodor 1640, Czudowski, Anna = Serebrysko 1640, Dołmatowicz-Isajkowski, Samuel 1658, Hański, Jakub 1635, Korczowski, Adam 1641, 1644, Lenkiewicz-Ipohorski, Aleksander 1644, Wyszpolski, Benedykt, Mikołaj 1645.

Uniates: Dachnowicz, Andrzej 1602, 1613, Dołmatowicz-Isajkowski, Dołmat 1656, Horain, Jan since 1634, Jerzy 1656, Kotłubaj-Iliaszewski, Michał $\dagger$ 1625, Sylwester 1628, Artemij 1645, Uhrynowski, Lazar 1646.

To complete the picture, it should be said that among immigrant Polish gentry representing the middle and lower economic levels, there were both Catholics and dissenters. However, there are only fragmentary data available, referring to the faith of the members of that group. Noteworthy, it's not only Catholics, like Bielecki, Bolanowski, Grzybowski, Kaniński, Pontkowski, Staniszewski, Sulimowski, Uniszowski, Węzłowski, Wilga, Zakrzewski, Ziemblicki, and Ziólkowski, who settled in the borderlands. There were also Arians (Arciszewski, Bieniewski, Czechowski, Nasiłowski, Przypkowski, Sowiński, Stan, Suchodolski) as well as Calvinists (Bogucki, Gamysz, Kochowski, Krupko-Przecławski, Rej, Pomsta).

\section{Translated by Kamil O. Kuraszkiewicz}

First published as: 'Struktura wyznaniowa szlachty kijowskiej 1569-1648', Odrodzenie i Reformacja $w$ Polsce, 48, 2004, pp. 199-220. 